MALAYA JOURNAL OF MATEMATIK

Malaya J. Mat. 9(04)(2021), 194-205.

http://doi.org/10.26637/mjm904/003

\title{
Disjunctive total domination in some tree networks
}

\author{
VECDİ AYTAÇ* ${ }^{* 1}$ AND TUfan TURACI ${ }^{2}$ \\ ${ }^{1}$ Faculty of Engineering, Department of Computer Engineering, Ege University, 35100 Izmir, Turkey. \\ 2 Faculty of Engineering, Department of Computer Engineering, Pamukkale University, 20160 Denizli, Turkey.
}

Received 12 June 2021; Accepted 28 August 2021

\begin{abstract}
Networking has been an essential field of multidisciplinary study, including computational theory, mathematics, social sciences, computer science, and other theoretical and applied sciences. The vulnerability determines the network's resistance to interruption of information flow after the breakdown of particular stations or transmission connections. Recently, new vulnerability parameter namely the disjunctive total domination number has been defined by Henning and Naicker [14]. This measure finds the critical vertices with an important position in the graph. In this context, we consider and compute exact formulae for the disjunctive total domination number in some tree networks.
\end{abstract}

AMS Subject Classifications: 05C69, 68M10, 68R10.

Keywords: Network Design and Communication, Complex Networks, Disjunctive total domination number, Trees.

\section{Contents}

1 Introduction

2 Disjunctive Total Domination Numbers of Some Trees

3 Conclusion

\section{Introduction}

The connected graph can model the network, the vertex in the graph represents a network node, and the edge represents a contact connection between the two nodes [7, 16]. There are many parameters in graph theory for network analysis and to determine some properties of the network. Graph theory techniques facilitate representation and analysis during a vulnerability assessment of complex networks. The theory is based on a set of measurements that evaluate networks and include graph vulnerability parameters. The proposed solutions for the network's vulnerability were rooted in the graph theoretical principles, especially the concepts of domination [19].

Theory of domination is one of the most important branches of graph vulnerability, which has wide application in network designings. It has a wide variety of uses in many areas, such as computer science, communication networks, transportation networks, biological and social networks, operations research, chemistry, economics, engineering, and applied mathematics; the principle of domination has recently become the center of graph theory research activity. This is largely due to a variety of new parameters that can be developed from the basic definition of domination $[10,14,15]$. Disjunctive total domination is the new domination parameter defined recently. Henning and Naicker [14] defined the disjunctive total domination as a relaxation of total domination.

A set $S \subseteq V(G)$ is a dominating set if every vertex in $V(G)-S$ is adjacent to at least one vertex in $S$. The minimum cardinality taken over all dominating sets of $G$ is called the domination number of $G$ and is denoted

*Corresponding author. Email address: vecdi.aytac@ege.edu.tr (Vecdi Aytaç), tturaci@pau.edu.tr (Tufan Turacı) 
Disjunctive total domination in some tree networks

by $\gamma(G)[3,10]$. A total dominating set, abbreviated a TD-set, of a graph $G$, with no isolated vertex is a set $S$ of vertices of $G$ such that every vertex in $V(G)$ is adjacent to at least one vertex in $S$. The total domination number of $G$, denoted by $\gamma_{t}(G)$, is the minimum cardinality of a TD-set of $G$ [3, 15]. Let $S \subseteq V(G)$. The set $S$ can be the disjunctive total dominating set of the graph $G$ if and only if it satisfies any of the following properties. For every vertex $v \in V(G)$;

(i) $v$ is adjacent to a vertex of $S$,

(ii) at least two vertices at a distance of 2 from the vertex $v$ must be in the set $S$.

The disjunctive total dominating set of the graph $G$ is briefly called DDT-set. The disjunctive total domination number of $G$ is the minimum cardinality of a DTD-set of $G$ and denoted by $\gamma_{t}^{d}(G)$. A DTD-set of cardinality $\gamma_{t}^{d}(G)$ is called a $\gamma_{t}^{d}(G)$-set. Clearly, every TD-set is a DTD-set, furthermore the result $\gamma_{t}^{d}(G) \leq \gamma_{t}(G)$ is obtained in [12-14]. This parameter is studied on grids, trees, permutation graphs, claw-free graphs, shadow distance graph of some special graphs and it is applied on some graph modifications such as bondage and subdivision [1, 2, 12-14].

We consider the disjunctive total domination number as a metric for network vulnerability. In this model, we find the critical vertices with an important position in the graph. Since disjunctive total domination number is considered to be a reasonable measure for the vulnerability of graphs, it is of particular interest to evaluate the disjunctive total domination number of different classes of graphs. Suppose one can break a more complex network into smaller networks, then under some conditions. In that case, the optimization problem's solutions on the smaller networks can be combined to solve the optimization problem on the larger network. Thus, calculation of the disjunctive total domination number for simple graph types is important.

For notation and graph theory terminology, we in general follow [10, 19]. Specifically, let $G=(V(G), E(G))$ be a simple undirected graph with vertex set $V(G)$ and edge set $E(G)$. The set of all adjacent vertices to vertex $v \in V(G)$ in $G$ is called neighborhood and denoted by $N_{G}(v)$ or $N(v)$. The close neighborhood of this vertex is defined as $N_{G}(v) \cup\{v\}$ and denoted by $N_{G}[v]$ or $N[v]$. The other basic parameter for graphs is the degree of vertex $v \in V(G)$, which is defined as the number of vertices in $N_{G}(v)$ and denoted by $\operatorname{deg}(v)$. Assume that the vertices $u$ and $v$ belong to the graph $G$. For these vertices, $d(u, v)$ is defined as the distance of the shortest path between these vertices.Furthermore, $\operatorname{diam}(G)$ is defined as the diameter of $G$, and it is the highest distance value within the vertices of $G . \Delta(G)=\max \{\operatorname{deg}(v) \mid v \in V(G)\}$ and $\delta(G)=\min \{\operatorname{deg}(v) \mid v \in V(G)\}$ represent the maximum and minimum degree, respectively. The vertex with $\operatorname{deg}(v)=1$ is said to be a pendant vertex or leaf vertex. The vertex adjacent to the pendant vertex is called the support vertex.

Now, we make use of the following known theorems in our results.

Theorem 1.1. [14] For $n \geq 3, \gamma_{t}^{d}\left(C_{n}\right)=\frac{2 n}{5}$ if $n \equiv 0(\bmod 5)$; and $\gamma_{t}^{d}\left(C_{n}\right)=\left\lceil\frac{2(n+1)}{5}\right\rceil$, otherwise.

Theorem 1.2. [14] For $n \geq 3, \gamma_{t}^{d}\left(P_{n}\right)=\left\lceil\frac{2(n+1)}{5}\right\rceil+1$ if $n \equiv 1(\bmod 5)$; and $\gamma_{t}^{d}\left(P_{n}\right)=\left\lceil\frac{2(n+1)}{5}\right\rceil$, otherwise.

Lemma 1.3. [14] If $v$ is a support vertex in a graph $G$ with exactly one neighbor $w$ that is not a leaf, then there is a $\gamma_{t}^{d}(G)$-set that contains $v$. Further if $\operatorname{deg}(w)=2$, then there is a $\gamma_{t}^{d}(G)$-set that contains both $v$ and $w$.

\section{Disjunctive Total Domination Numbers of Some Trees}

In this section, the distinctive total dominance numbers of certain tree-type networks such as the double comet graph, the double star graph, the comet graph, the generalized caterpillars, the comb graph, the thorn graph $P_{n}^{*}$, the binomial tree and the complete $k$-ary tree are computed and exact formulae are presented.

Definition 2.1. [8] The double star graph $S(x, y)$, where $x, y \geq 0$, is the graph consisting of the union of two star graphs $K_{1, x}$ and $K_{1, y}$ together with an edge joining their centers.

Theorem 2.2. If $G \cong S(x, y)$ of order $x+y$, where $x, y \geq 0$, then, $\gamma_{t}^{d}(G)=2$. 


\section{Vecdi AYTAÇ and Tufan TURACI}

Proof. Note that $V(G)=V\left(K_{1, x}\right) \cup V\left(K_{1, y}\right)$. Furthermore, let $u_{1}$ and $u_{2}$ be the central vertices of $K_{1, x}$ and $K_{1, y}$, respectively. It is easily seen that $\operatorname{deg}\left(x_{i}\right)=\operatorname{deg}\left(y_{i}\right)=1$ for every vertices $x_{i}$ and $y_{i}$, where $x_{i} \in V\left(K_{1, x}\right)$ and $y_{i} \in V\left(K_{1, y}\right), \operatorname{deg}\left(u_{1}\right)=x+1$ and $\operatorname{deg}\left(u_{2}\right)=y+1$. If a DTD-set of $G$ is considered $S$, then taking $u_{1}$ and $u_{2}$ to the set $S$ yields $\gamma_{t}^{d}(G) \leq 2$. Furthermore, we have $\gamma_{t}^{d}(G) \geq 2$ for any graph $G$ by the definition of disjunctive total domination number. So, $\gamma_{t}^{d}(G) \geq 2$. As a consequence, by combining the lower and upper bounds, we obtain $\gamma_{t}^{d}(G)=2$.

Definition 2.3. [4] The comet graph $C(t, r)$ is the graph obtained by identifying one end of the path $P_{t}$ with the center of the star graph $K_{1, r}$. This graph is illustrated in Figure 1.

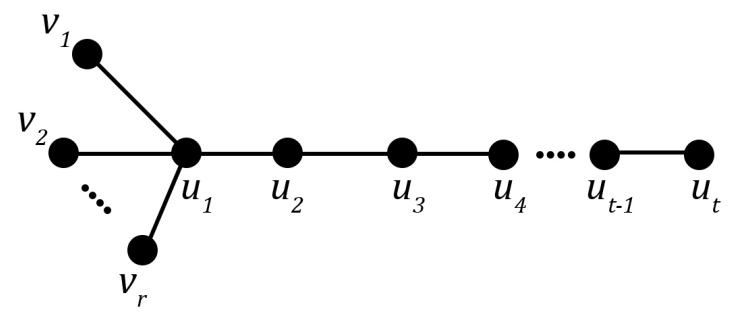

Figure 1: The comet graph $C(t, r)$.

Theorem 2.4. If $G \cong C(t, r)$ of order $t+r$, then $\gamma_{t}^{d}(G)= \begin{cases}\left\lceil\frac{2(t+1)}{5}\right\rceil+1, \text { if } t \equiv 0,1,4(\bmod 5) \text {; } \\ \left\lceil\frac{2(t+1)}{5}\right\rceil, & \text {, otherwise. }\end{cases}$

Proof. Note that $V(G)=V\left(P_{t-1}\right) \cup V\left(K_{1, r}\right)$. Furthermore, $V\left(P_{t-1}\right)=\left\{u_{2}, u_{3}, \ldots, u_{t}\right\}$ and $V\left(K_{1, r}\right)=$ $\left\{u_{1}, v_{1}, \ldots, v_{r}\right\}$, where $u_{1}$ is the center vertex. Suppose $S$ is a DTD-set in $G$. By Lemma 1.3, $u_{1}$ must be in $S$. Thus, all vertices $v_{i}$ and $u_{2}$ are disjunctively totally dominated by the vertex $u_{1}$. The disjunctive total undominated vertices by $S$ are the vertices of the path graph with $(t-1)$ vertices. So, the rest of the proof has to be made similar to the proof of Theorem 1.2. In this case, if $t \equiv 0,1,4(\bmod d 5)$, then $\gamma_{t}^{d}(G)=|S|=\lceil 2(t+1) / 5\rceil+1$, while if $t \equiv 2,3(\bmod 5)$, then $\gamma_{t}^{d}(G)=|S|=\lceil 2(t+1) / 5\rceil$ are obtained.

Definition 2.5. [5] The graph obtained by adding $x$ and $y$ vertices, which are pendant, to the end vertices of the path graph with $n-x-y$ vertices is called double comet $D C(n, x, y)$. For $x, y \geq 1$ and $n \geq x+y+2$ the double comet $D C(n, x, y)$ is one of the tree graphs. $D C(n, x, y)$ is a graph with $n$ vertices, $x+y$ of which is leaves. This graph is illustrated in Figure 2.

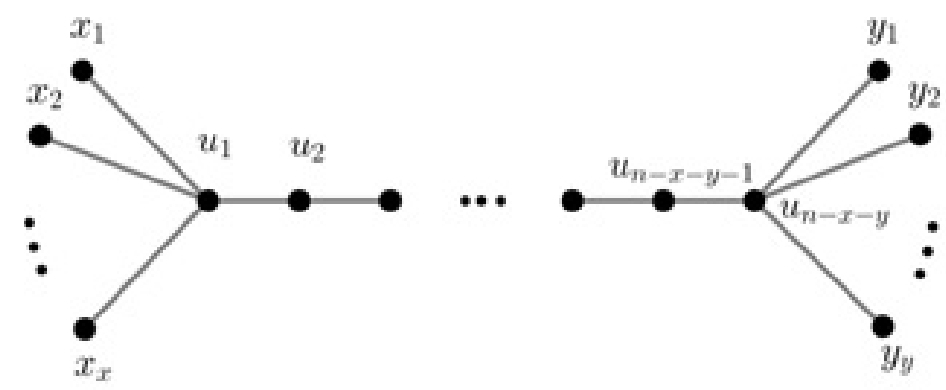

Figure 2: The double comet graph $D C(n, x, y)$. 
Disjunctive total domination in some tree networks

Theorem 2.6. If $G \cong D C(n, x, y)$ with $x, y \geq 2$ and $n \geq x+y+3$, then

$$
\gamma_{t}^{d}(G)=\left\{\begin{array}{l}
\left\lceil\frac{2(n-x-y+1)}{5}\right\rceil+2, \text { if }(n-x-y) \equiv 4(\bmod 5) \\
\left\lceil\frac{2(n-x-y+1)}{5}\right\rceil, \text { if }(n-x-y) \equiv 2(\bmod 5) \\
\left\lceil\frac{2(n-x-y+1)}{5}\right\rceil+1, \text { otherwise. }
\end{array}\right.
$$

Proof. Note that $V(G)=V\left(P_{n-x-y-2}\right) \cup V\left(K_{1, x}\right) \cup V\left(K_{1, y}\right) \quad$ in $\quad$ which $V\left(P_{n-x-y-2}\right)=\left\{u_{2}, u_{3}, \ldots, u_{n-x-y-1}\right\}, \quad V\left(K_{1, x}\right) \stackrel{=}{=} \quad\left\{u_{1}, x_{1}, x_{2} \ldots, x_{x}\right\} \quad$ and $V\left(K_{1, y}\right)=\left\{u_{n-x-y}, y_{1}, y_{2} \ldots, y_{y}\right\}$. Suppose $S$ is a DTD-set in $G$. By Lemma 1.3, the vertices $u_{1}$ and $u_{n-x-y}$ must be taken to the set $S$. Thus the vertices not disjunctively totally dominated by the set $S$ form the path graph. As in the proof of Theorem 1.2, the construction of the set $S$ is continued. In this case, if $(n-x-y) \equiv 4(\bmod 5)$, then $\gamma_{t}^{d}(G)=|S|=\lceil(2(n-x-y+1) / 5)\rceil+2 ;$ if $(n-x-y) \equiv 2(\bmod 5)$, then $\gamma_{t}^{d}(G)=|S|=\lceil(2(n-x-y+1) / 5)\rceil$ and otherwise $\gamma_{t}^{d}(G)=|S|=\lceil(2(n-x-y+1) / 5)\rceil+1$ are obtained.

Definition 2.7. [17] The graph obtained by joining a pendant edge at each vertex of a path $P_{n}$ is called a comb graph and is denoted by $P_{n} \square K_{1}$. The graph $P_{5} \square K_{1}$ is illustrated in Figure 3.

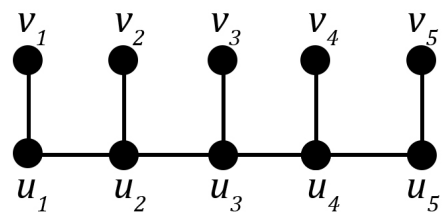

Figure 3: The comb graph $P_{5} \square K_{1}$.

Theorem 2.8. If $G \cong P_{n} \square K_{1}$ of order $2 n$, then $\gamma_{t}^{d}(G)=4+\lfloor(n-4) / 2\rfloor$.

Proof. Note that $V(G)=\left\{u_{i}, v_{i} \mid 1 \leq i \leq n\right\}$ and $E(G)=\left\{u_{i} v_{i} \mid 1 \leq i \leq n\right\} \cup\left\{u_{i} u_{i+1} \mid 1 \leq i \leq n-1\right\}$. It is obvious that $|V(G)|=2 n,|E(G)|=2 n-1, \operatorname{deg}\left(v_{i}\right)=1$ where $i \in\{1, \ldots, n\}, \operatorname{deg}\left(u_{1}\right)=\operatorname{deg}\left(u_{n}\right)=2$ and $\operatorname{deg}\left(u_{i}\right)=3$ where $i \in\{2, \ldots, n-1\}$. We set the upper limits to the disjunctive total domination number of $G$, first. Suppose $D$ is a DTD-set in $G$. According to degree of vertices of $G$, some $u_{i}$-vertices $(i \in\{2, \ldots, n-1\})$ must be taken to the set $D$. To disjunctively totally dominate the vertices $v_{1}$ and $v_{n}$, it must be $\left\{u_{1}, u_{n}\right\} \subseteq D$. Since $N_{G}\left(u_{1}\right)=\left\{v_{1}, u_{2}\right\}$ and $N_{G}\left(u_{n}\right)=\left\{v_{n}, u_{n-1}\right\},\left\{u_{2}, u_{n-1}\right\} \subset D$ should be to disjunctively totally dominate the vertices $u_{1}$ and $u_{n}$. Thus, the set $D$ is as follows:

$$
D=\bigcup_{i=0}^{\left\lfloor\frac{n-4}{2}\right\rfloor+1}\left\{u_{2 i+4}\right\} \cup\left\{u_{1}, u_{2}, u_{n-1}, u_{n}\right\} .
$$

Clearly, the set $D$ is a DTD-set for every $n \geq 5$. Furthermore, we get $|D|=4+\lfloor(n-4) / 2\rfloor$, also is an upper bound. Thus, $\gamma_{t}^{d}(G) \leq 4+\lfloor(n-4) / 2\rfloor$ is obtained.

To prove the inverse of equality, let the set $T$ be a $\gamma_{t}^{d}(G)$-set of $G$. Assume that the two vertices are adjacent in $T$. Furthermore, the set $S$ is as follows:

$$
S=\bigcup_{i=0}^{\left\lfloor\frac{n}{3}\right\rfloor-1}\left\{u_{3 i+1}, u_{3 i+2}\right\}
$$




\section{Vecdi AYTAÇ and Tufan TURACI}

, where $S \subseteq T$. If $n \equiv 1(\bmod 3)$, we have $T=S \cup\left\{v_{n-1}, v_{n}\right\}$; if $n \equiv 0(\bmod 3)$, we have $T=S \cup\left\{v_{n}\right\}$ and if $n \equiv 2(\bmod 3)$, we have $T=S$. Thus, we obtain $|T|=2\lfloor n / 3\rfloor+2$ for $n \equiv 1(\bmod 3),|T|=2\lfloor n / 3\rfloor+1$ for $n \equiv 0(\bmod 3)$ and $|T|=2\lfloor n / 3\rfloor$ for $n \equiv 2(\bmod 3)$. These results contradict the previous upper bound for $n \geq 7$.

Furthermore, we get $\left\{u_{1}, u_{2}, u_{n-1}, u_{n}\right\} \subset T$. However, apart from these vertices, no two vertices in $T$ should be adjacent to each other. If the distance between the two vertices is at least three, all vertices in $G$ cannot be disjunctively totally dominated. So, the distance between two vertices must be exactly 2 . Thus, it is easy to see that $\gamma_{t}^{d}(G) \geq 4+\lfloor(n-4) / 2\rfloor$, also we have $\gamma_{t}^{d}(G)=4+\lfloor(n-4) / 2\rfloor$.

Corollary 2.9. If $G \cong P_{n} \square K_{1}$ of order $2 n$, then $\gamma_{t}^{d}(G)=4+\gamma\left(P_{n-4}\right)$.

Definition 2.10. [9] Let $p_{1}, p_{2}, \ldots, p_{n}$ be non-negative integers and the graph $G$ be such a graph, where $|V(G)|=n$. The thorn graph of the graph $G$ with parameters $p_{1}, p_{2}, \ldots, p_{n}$ is obtained by attaching $p_{i}$ new vertices of degree one to the vertex $u_{i}$ of the graph $G$, where $i=\overline{1, n}$. The thorn graph of the graph $G$ will be denoted by $G^{*}$ or if the respective parameters need to be specified, by $G^{*}\left(p_{1}, p_{2}, \ldots, p_{n}\right)$. The graph $P_{7}^{*}(2,1,1,3,2,1,4)$ is illustrated in Figure 4.

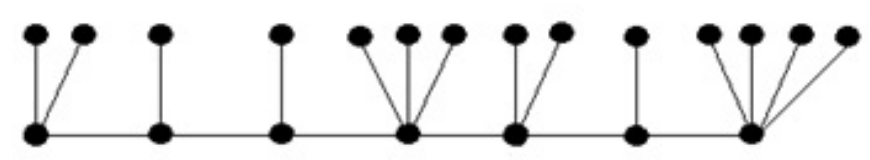

Figure 4: The thorn graph $P_{7}^{*}(2,1,1,3,2,1,4)$.

Theorem 2.11. If $G \cong P_{n}^{*}$ is a thorn graph of $P_{n}$ with $p_{i} \geq 2$, then $\gamma_{t}^{d}(G)=\gamma_{t}^{d}\left(P_{n} \square K_{1}\right)$.

Proof. The proof is quite close to that of Theorem 2.8, so we omit it.

Definition 2.12. [18] $C_{(t, 0)} P_{n}$ is a generalized Caterpillar obtained from the path graph $P_{n}$ by attaching $t$ vertices of degree one to each vertex of degree two of $P_{n}$. The tree $C_{(t, 1)} P_{n}$ is a generalized Caterpillar obtained from the path graph $P_{n}$ by attaching $m$ vertices of degree two to each vertex of degree two of $P_{n}$. The graph $C_{(3,0)} P_{7}$ and $C_{(3,1)} P_{7}$ are illustrated in Figure 5.

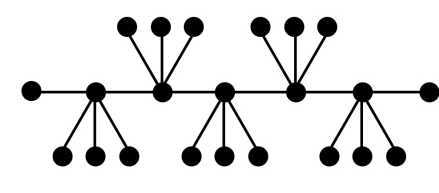

(a)

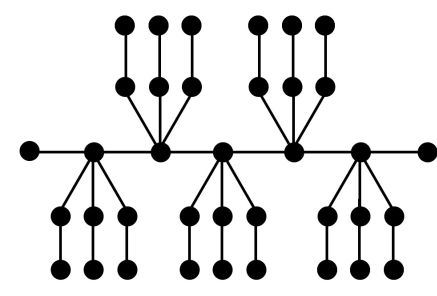

(b)

Figure 5: (a) The graph $C_{(3,0)} P_{7}$ and (b) the graph $C_{(3,1)} P_{7}$.

Theorem 2.13. Let $G \cong C_{(t, 0)} P_{n}$ be generalized caterpillar graph with $(n+t(n-2))$-vertices. Then, for $t \geq 2$ and $p_{i}=t, \gamma_{t}^{d}(G)=\gamma_{t}^{d}\left(P_{n-2}^{*}\right)$.

Proof. The proof is quite close to that of Theorem 2.8, so we omit it. 
Disjunctive total domination in some tree networks

Theorem 2.14. Let $G \cong C_{(t, 1)} P_{n}$ be generalized caterpillar graph with $(n+t(n-2))$-vertices. Then, for $t \geq 3$, $\gamma_{t}^{d}(G)=t(n-2)$.

Proof. The graph $G$ has $(n+t(n-2))$-vertices. Let $V(G)=V_{1} \cup V_{2} \cup V_{3}$, where $V_{1}=\left\{u_{i} \in V\left(P_{n}\right) \mid 1 \leq i \leq n\right\}$, $V_{2}=\left\{v_{i} \in\left(V(G)-V\left(P_{n}\right)\right) \mid \operatorname{deg}\left(v_{i}\right)=2\right.$ and $\left.1 \leq i \leq t(n-2)\right\}$ and $V_{3}=\left\{w_{i} \in\left(V(G)-V\left(P_{n}\right)\right) \mid \operatorname{deg}\left(w_{i}\right)=\right.$ 1 and $1 \leq i \leq t(n-2)\}$. Clearly, we have $\operatorname{deg}\left(u_{1}\right)=\operatorname{deg}\left(u_{n}\right)=1$ and $\operatorname{deg}\left(u_{i}\right)=2$ for the vertices of $V_{1}$, where $i \in\{2, \ldots, n-1\}$. Suppose $S$ is a $\gamma_{t}^{d}(G)$-set of $G$. Since $\operatorname{deg}\left(w_{i}\right)=1$ for each vertex $w_{i} \in V_{3}$, the all vertices of $V_{2}$ must be taken to the set $S$. Therefore, each vertex of $V_{1}$ is disjunctively totally dominated by the set $S$. Thus, all vertices in $G$ are disjunctively totally dominated by $S$. It is easily seen that the set $S$ is unique and there is no other set of $\gamma_{t}^{d}(G)$-set. Note that $\left|V_{2}\right|=t(n-2)$. Then, $|S|=t(n-2)$. Hence, we get $\gamma_{t}^{d}(G)=t(n-2)$. Thus, the proof holds.

Definition 2.15. [6] The binomial tree $B_{n}$ with root $R$ is the tree defines as follows:

i. If $n=0$, then $B_{n}=B_{0}=R$, i.e., the binomial tree of order zero consists of a single root $R$.

ii. If $n>0$, then $B_{n}=R, B_{0}, B_{1}, \ldots, B_{n-1}$, i.e., the binomial tree of order $n>0$ comprises the root $R$ and $n$ binomial subtrees $B_{0}, B_{1}, \ldots, B_{n-1}$.

In Figures 6 and 7 , the binomial trees $B_{4}$ and $B_{5}$ are illustrated.

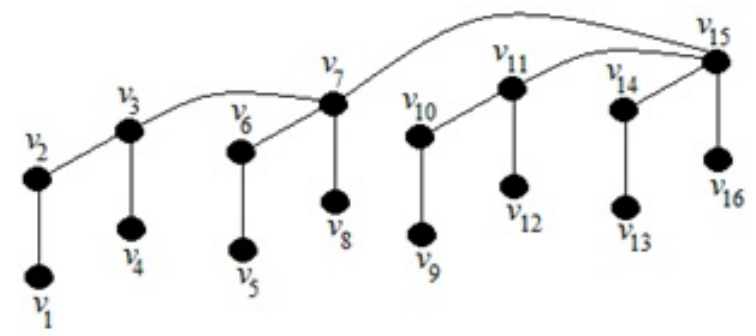

Figure 6: The binomial tree $B_{4}$.

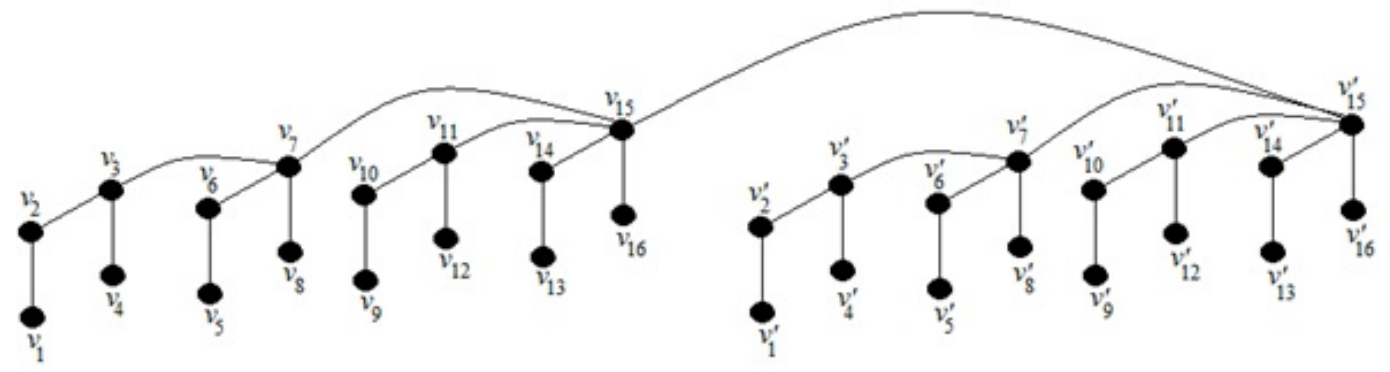

Figure 7: The binomial tree $B_{5}$.

Theorem 2.16. If $G \cong B_{n}$ of order $2^{n}$ with $n \geq 5$, then $\gamma_{t}^{d}(G)=7\left(2^{n-4}\right)$.

Proof. The binomial tree $B_{n}$ has $2^{n}$-vertices. It is clear that $\gamma_{t}^{d}\left(B_{0}\right)=1, \gamma_{t}^{d}\left(B_{1}\right)=\gamma_{t}^{d}\left(B_{2}\right)=2$, and $\gamma_{t}^{d}\left(B_{3}\right)=$ 4 for $n \leq 3$. Let $n=4$, and let $S_{1}$ be a $\gamma_{t}^{d}(G)$-set of $B_{4}$. It is easily seen that $S_{1}=\left\{v_{2}, v_{3}, v_{6}, v_{10}, v_{11}, v_{14}, v_{15}\right\}$ (see Figure 6). Then, we get $\gamma_{t}^{d}\left(B_{4}\right)=7$.

Suppose $S$ is a $\gamma_{t}^{d}(G)$-set of $B_{5}$. Since $B_{5}$ has two copies of $B_{4}$, the vertices $\left\{v_{2}, v_{3}, v_{6}, v_{10}, v_{11}, v_{14}, v_{15}\right\}$ and $\left\{v_{2}^{\prime}, v_{3}^{\prime}, v_{6}^{\prime}, v_{10}^{\prime}, v_{11}^{\prime}, v_{14}^{\prime}, v_{15}^{\prime}\right\}$ in the first and second copies of $B_{4}$, respectively, must be taken to the set $S$ 


\section{Vecdi AYTAÇ and Tufan TURACI}

(see Figure 7). Thus, we have $S=\left\{v_{2}, v_{3}, v_{6}, v_{10}, v_{11}, v_{14}, v_{15}, v_{2}^{\prime}, v_{3}^{\prime}, v_{6}^{\prime}, v_{10}^{\prime}, v_{11}^{\prime}, v_{14}^{\prime}, v_{15}^{\prime}\right\}$. Then, we obtain $\gamma_{t}^{d}\left(B_{5}\right)=2 \gamma_{t}^{d}\left(B_{4}\right)=14$. With the same thought, $\gamma_{t}^{d}\left(B_{6}\right)=2 \gamma_{t}^{d}\left(B_{5}\right)=28$ and $\gamma_{t}^{d}\left(B_{7}\right)=2 \gamma_{t}^{d}\left(B_{6}\right)=56$ are obtained. If this continue for $n \geq 5$, we get the following recurrence formula:

$$
\gamma_{t}^{d}\left(B_{n}\right)=2 \gamma_{t}^{d}\left(B_{n-1}\right) \text { for } n \geq 5 .
$$

From this formula, we have:

$$
\gamma_{t}^{d}\left(B_{n}\right)=2 \gamma_{t}^{d}\left(B_{n-1}\right)=2\left(2 \gamma_{t}^{d}\left(B_{n-2}\right)\right)=2^{2} \gamma_{t}^{d}\left(B_{n-1}\right)=\ldots=2^{n-4} \gamma_{t}^{d}\left(B_{4}\right)
$$

Furthermore, we obtain

$$
\gamma_{t}^{d}\left(B_{n}\right)=2^{i} \gamma_{t}^{d}\left(B_{n-i}\right), i \in\{1,2, n-1\} .
$$

This equality can be seen by the induction method.

Let $i=1$.

Thus, we have $\gamma_{t}^{d}\left(B_{n}\right)=2 \gamma_{t}^{d}\left(B_{n-1}\right)$, also is clear by Eq. (1). We prove this statement with induction on $i$. When $i=1$, we have $\gamma_{t}^{d}\left(B_{n}\right)=2^{i} \gamma_{t}^{d}\left(B_{n-i}\right)$ and via the Eq. (1), this is valid. We suppose that the result is true for $i=k$ and prove it for $i=k+1$. By induction hypothesis and Eq. (1), we get

$$
\gamma_{t}^{d}\left(B_{n}\right)=2^{k} \gamma_{t}^{d}\left(B_{n-k}\right)=2^{k}\left(2 \gamma_{t}^{d}\left(B_{n-k-1}\right)\right)=2^{k+1} \gamma_{t}^{d}\left(B_{n-k-1}\right) .
$$

This means the claim is valid where $i=k+1$. Hence, we get

$$
\begin{aligned}
& \gamma_{t}^{d}\left(B_{n}\right)=2^{i} \gamma_{t}^{d}\left(B_{n-i}\right), \text { where } 1 \leq i \leq n-4, \\
& \gamma_{t}^{d}\left(B_{n}\right)=7 .
\end{aligned}
$$

Since the first case obtained when $i=n-4$ is $n=4$, the following is obtained from Eq. (2).

$$
\gamma_{t}^{d}\left(B_{n}\right)=2^{n-4}\left(B_{n-(n-4)}\right)=2^{n-4}\left(\gamma_{t}^{d}\left(B_{4}\right)\right)=7\left(2^{n-4}\right) \text {. }
$$

Definition 2.17. [6] The complete k-ary tree of height $h, T_{h}^{k}$, is a rooted tree with each leaf having the same depth and each vertex except the leaves in degree $k . T_{h}^{k}$ in which every non-leaf vertex has exactly $k$-children and the distance from the root to each leaf is exactly $h$. The complete $k$-ary tree for $k \geq 2$ has $\frac{k^{h+1}-1}{k-1}$ vertices and $\frac{k^{h+1}-1}{k-1}-1=\frac{k^{h+1}-k}{k-1}$ edges. The complete binary tree is the complete $k$-ary tree with $k=2$. Figure 8 shows an example of a complete $k$-ary tree $T_{4}^{2}(k=2$ and $h=4)$. In Figure 8 , the value of $L$ expresses the depth of each vertex.

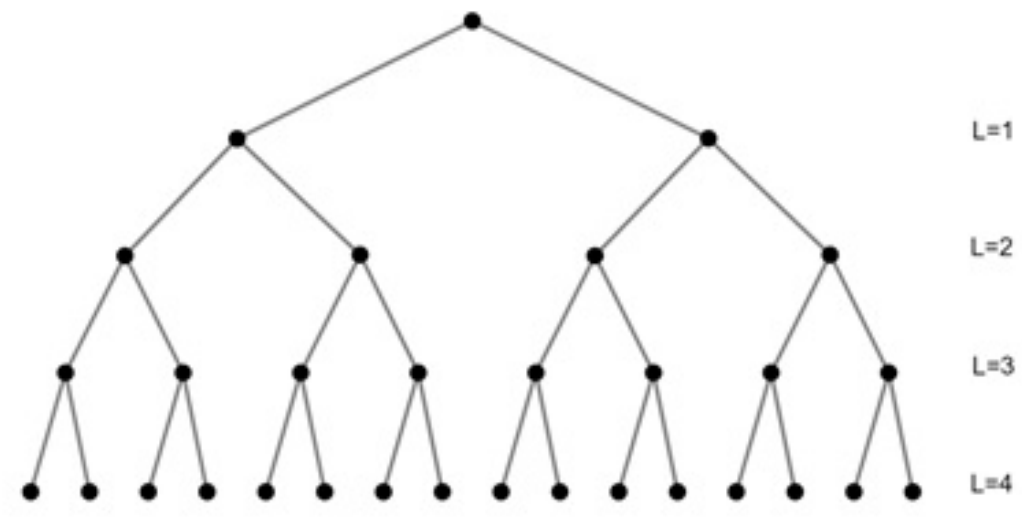

Figure 8: The complete 2-ary tree $T_{4}^{2}(k=2$ and $h=4)$. 
Disjunctive total domination in some tree networks

Theorem 2.18. If $G \cong T_{h}^{k}$ of order $\frac{k^{h+1}-1}{k-1}$, where $h \geq 4$ and $k \geq 3$, then

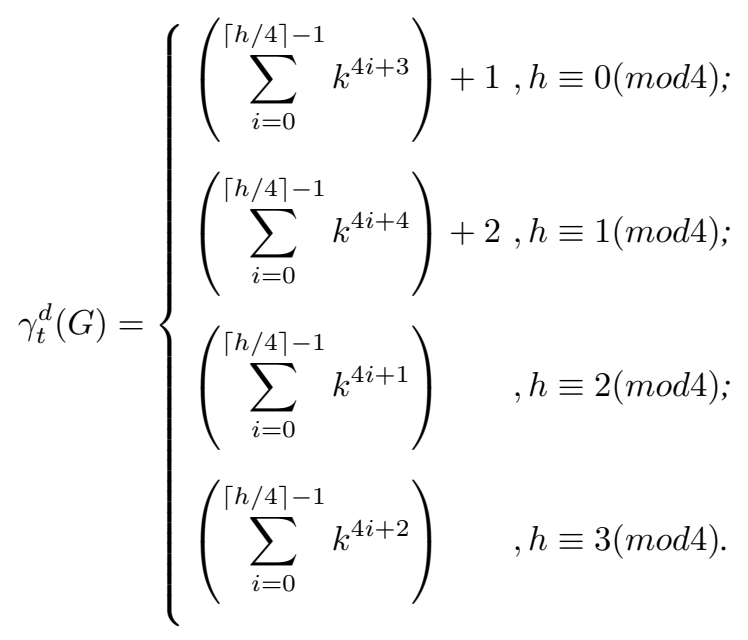

Proof. By the definition of complete $k$-ary tree $T_{h}^{k}$, we know that there are $k^{0}, k^{1}, \ldots, k^{h}$ vertices in the levels $0^{t h}, 1^{t h}, \ldots, h^{t h}$, respectively. Suppose $S$ is a DTD-set in $T_{h}^{k}$ for $h \geq 4$ and $k \geq 3$. We know that the vertices that in the level $h^{\text {th }}$ are called leaf vertices. Since the degree of each leaf vertex is 1 , the set $S$ must include support vertices in the level $(h-1)^{t h}$. Furthermore, the distance between each vertex in $S$ and at least two vertices in $S$ is two. Thus, the vertices which are in the levels $h^{t h},(h-2)^{t h}$ and $(h-3)^{t h}$ are disjunctively totally dominated by the set $S$. Then, the vertices that in the level $(h-5)^{t h}$ must be added to the set $S$. It is easy to see that distance of the any two vertices which are in distinct levels is four. With the same thought, the set will be occurred. But, we have four cases according to $h$.

Case 1. $h \equiv 0(\bmod 4)$.

$$
\lceil h / 4\rceil-1
$$

Let $L=\sum_{i=0}\{4 i+3\}$, where the elements of $L$ is the levels of tree $T_{h}^{k}$. Let the set $S$ includes the vertices which are in the levels in $L$. Thus, we get $|S|=\sum_{i=0}^{\lceil h / 4\rceil-1} k^{4 i+3}$. But the root vertex of $T_{h}^{k}$ is not disjunctively totally dominated by $S$. If any vertex in the first level is added to $S$, then root vertex is disjunctively totally dominated. So, $\gamma_{t}^{d}\left(T_{h}^{k}\right)=|S|=\left(\sum_{i=0}^{\lceil h / 4\rceil-1} k^{4 i+3}\right)+1$ is obtained.

Case 2. $h \equiv 1(\bmod 4)$.

Let $L=\sum_{i=0}^{\lceil h / 4\rceil-1}\{4 i+4\}$, where the elements of $L$ is the levels of tree $T_{h}^{k}$. Let the set $S$ includes the vertices which are in the levels in $L$. Thus, we get $|S|=\sum_{i=0}^{\lceil h / 4\rceil-1} k^{4 i+4}$. But the root vertex and the vertices which are in first level of $T_{h}^{k}$ are not disjunctively totally dominated by $S$. If any vertex in the first level and the root vertex are added to $S$, then all vertices of $T_{h}^{k}$ are disjunctively totally dominated by $S$. So, $\gamma_{t}^{d}\left(T_{h}^{k}\right)=|S|=\left(\sum_{i=0}^{\lceil h / 4\rceil-1} k^{4 i+4}\right)+2$ is obtained. 


\section{Vecdi AYTAÇ and Tufan TURACI}

Case 3. $h \equiv 2(\bmod 4)$.

$$
\lceil h / 4\rceil-1
$$

Let $L=\sum_{i=0}\{4 i+1\}$, where the elements of $L$ is the levels of tree $T_{h}^{k}$. Let the set $S$ includes the vertices which are in the levels in $L$. Thus, we get $|S|=\sum_{i=0}^{\lceil h / 4\rceil-1} k^{4 i+1}$. Clearly, all vertices of $T_{h}^{k}$ are disjunctively totally dominated by $S$. So, $\gamma_{t}^{d}\left(T_{h}^{k}\right)=|S|=\sum_{i=0}^{\lceil h / 4\rceil-1} k^{4 i+1}$ is obtained.

Case 4. $h \equiv 3(\bmod 4)$.

Let $L=\sum_{i=0}^{\lceil h / 4\rceil-1}\{4 i+2\}$, where the elements of $L$ is the levels of tree $T_{h}^{k}$. This case is similar to the Case 3 . So, $\gamma_{t}^{d}\left(T_{h}^{k}\right)=|S|=\sum_{i=0}^{\lceil h / 4\rceil-1} k^{4 i+2}$ is obtained.

Thus, the proof of theorem is completed by the Cases 1,2, 3 and 4.

Theorem 2.19. If $G \cong T_{h}^{k}$ of order $\frac{k^{h+1}-1}{k-1}$, where $h \leq 3$ and $k \geq 3$, then $\gamma_{t}^{d}(G)=k^{h-1}$.

Proof. Suppose $S$ is a DTD-set in $T_{h}^{k}$. Due to leaf vertices of $T_{h}^{k}$, the all support vertices must be taken to the set $S$. It is clear that all vertices of $T_{h}^{k}$ are disjunctively totally dominated by $S$. So, we get $\gamma_{t}^{d}\left(T_{h}^{k}\right)=k^{h-1}$.

Theorem 2.20. If $G \cong T_{h}^{2}$ is a complete 2 -ary tree of order $2^{h+1}-1$, where $h \geq 4$, then

$$
\gamma_{t}^{d}(G)=\left\{\begin{array}{cl}
\frac{2\left(2^{h}-1\right)}{3} & , \text { if } t \equiv 0(\bmod 2) \\
2^{h-1}+2^{h-3} & , \text { otherwise. }
\end{array}\right.
$$

Proof. By the definition of complete $k$-ary tree $T_{h}^{k}$, we know that $T_{h}^{2}$ consists of 2 copies of $T_{h-1}^{2}$, also $T_{h-1}^{2}$ consists of 2 copies of $T_{h-2}^{2}$, etc. Clearly, we get $T_{1}^{2} \cong S_{1,2}$, where $S_{1,2}$ is a star graph. It is easily seen that $\gamma_{t}^{d}\left(T_{1}^{2}\right)=2$. Let $D$ be a $\gamma_{t}^{d}(G)$-set of $T_{h}^{2}$ for $h \leq 3$. To be disjunctively totally dominated each vertex of $T_{h}^{2}$, all vertices which are in $(h-1)^{t h}$ level must be added to the set $D$. Thus, all vertices except the vertices in $D$ are disjunctively totally dominated. Therefore, the vertex which in level zero must be added to $D$. So, all vertices of $T_{h}^{2}$ are disjunctively totally dominated by the set $D$. Clearly, $|D|=2^{h-1}+1$, also the set $D$ is the minimum DTD-set. As a result, $\gamma_{t}^{d}\left(T_{h}^{2}\right)=2^{h-1}+1$ for $h \leq 3$.

Let $h \geq 4$, and let $S_{1}$ be a $\gamma_{t}^{d}(G)$-set of $T_{4}^{2}$. Clearly, the set $S_{1}$ has the vertices of $v_{1}, v_{2}, v_{i}$, where $i \in\{7,8, \ldots, 14\}$ in the Figure 8. So, all vertices in $T_{4}^{2}$ and $S_{1}$ are disjunctively totally dominated by $S_{1}$. It is easy to see that $\left|S_{1}\right|=2^{3}+2=10$. Hence, $\gamma_{t}^{d}\left(T_{4}^{2}\right)=10$. Let $S$ be $\gamma_{t}^{d}(G)$-set of $T_{5}^{2}$. Since the tree $T_{5}^{2}$ has $k$-copies of $T_{4}^{2}$, all vertices which are correspond to vertices of $S_{1}$ in all copies of $T_{4}^{2}$ must be added to the set $S$. That is, $S=\bigcup_{j=1}^{2} S_{1}$. Thus, we get $|S|=2\left|S_{1}\right|$. So, $\gamma_{t}^{d}\left(T_{5}^{2}\right)=2\left(\gamma_{t}^{d}\left(T_{4}^{2}\right)\right)=2^{4}+2^{2}$ is obtained.

With the same thought, we consider the tree $T_{6}^{2}$. If the vertices of $\gamma_{t}^{d}(G)$-set of the tree $T_{5}^{2}$ which is copy of $T_{6}^{2}$, all vertices of $T_{6}^{2}$ are not disjunctively totally dominated. Because, the vertices which are in the DTD-set are not dominated. To disjunctively total dominate of these vertices, the vertices which are in 1-level must be taken in to the $\gamma_{t}^{d}(G)$-set. Thus, we obtain $\gamma_{t}^{d}\left(T_{6}^{2}\right)=2\left(\gamma_{t}^{d}\left(T_{5}^{2}\right)\right)+2$. Furthermore, we get following recursive formulas for $\gamma_{t}^{d}\left(T_{h}^{2}\right)$, where $h \geq 5$ : 
Disjunctive total domination in some tree networks

$$
\gamma_{t}^{d}\left(T_{h}^{2}\right)= \begin{cases}2 \gamma_{t}^{d}\left(T_{h-1}^{2}\right)+2 & , \text { if } h \equiv 0(\bmod 2) \\ \gamma_{t}^{d}\left(T_{h-1}^{2}\right) & , \text { otherwise. }\end{cases}
$$

Let $h=2 k+1$, where $k \in Z^{+}$. Then we have

$$
\gamma_{t}^{d}\left(T_{h}^{2}\right)=2 \gamma_{t}^{d}\left(T_{h-1}^{2}\right)=2\left(2 \gamma_{t}^{d}\left(T_{h-2}^{2}\right)\right)=2^{2} \gamma_{t}^{d}\left(T_{h-2}^{2}\right)=\ldots=2^{n-4}\left(T_{4}^{2}\right) .
$$

Let $h=2 k$, where $k \in Z^{+}$. Then we have

$$
\begin{aligned}
\gamma_{t}^{d}\left(T_{h}^{2}\right) & =2 \gamma_{t}^{d}\left(T_{h-1}^{2}\right)+2 \\
& =2\left(2 \gamma_{t}^{d}\left(T_{h-2}^{2}\right)+2\right)=2^{2}\left(\gamma_{t}^{d}\left(T_{h-2}^{2}\right)\right)+2 \\
& =2^{2}\left(2 \gamma_{t}^{d}\left(T_{h-3}^{2}\right)+2=2^{3}\left(\gamma_{t}^{d}\left(T_{h-3}^{2}\right)\right)+2^{3}+2\right. \\
& \cdot \\
& \cdot \\
& \cdot \\
& =2^{h-4} \gamma_{t}^{d}\left(T_{4}^{2}\right)+2^{h-5}+2^{h-7}+\ldots+2^{h-(h-3)}+2^{h-(h-1)} .
\end{aligned}
$$

Clearly, we get following result for $1 \leq i \leq h-4$.

$$
\begin{aligned}
& \gamma_{t}^{d}\left(T_{h}^{2}\right)=2^{i} \gamma_{t}^{d}\left(T_{h-i}^{2}\right), \text { if } h=2 k+1 \\
& \gamma_{t}^{d}\left(T_{h}^{2}\right)=2^{i} \gamma_{t}^{d}\left(T_{h-i}^{2}\right)+\sum_{i=0}^{\frac{h-4}{2}-1} 2^{2 i+1}, \text { if } h=2 k .
\end{aligned}
$$

If we use geometric series for $h=2 k$, then we have $\gamma_{t}^{d}\left(T_{h}^{2}\right)=2^{i} \gamma_{t}^{d}\left(T_{h-i}^{2}\right)+2\left(\frac{2^{i}-1}{2^{2}-1}\right)$.

These equalities can be proved by induction method, also remaining of the proof is similar to the proof of Theorem 2.16. So, the remaining of proof is omitted. Thus, the proof holds.

\section{Conclusion}

Various measures to determine the network robustness were suggested in the literature, and a number of graphtheoretical parameters were used to assess network reliability. We have discussed the disjunctive total domination number for some tree networks in this work. Suppose one can break a more complex network into smaller networks, then under some conditions. In that case, the optimization problem's solutions on the smaller networks can be combined to solve the optimization problem on the larger network. Thus, calculation of the disjunctive total domination number for simple graph types is important. 


\section{Vecdi AYTAÇ and Tufan TURACI}

\section{References}

[1] C. Çıfтçı, Disjunctive Total Domination of Some Shadow Distance Graphs, Journal of Mathematics and Applications, 3(2)(2020), 185-193.

[2] C. Çiftçi And V. AYtaÇ, Disjunctive total domination subdivision number of graphs, Fund. Inform., 174(1)(2020), 15-26.

[3] C. Çıftçı And A. AytaÇ, Porous Exponential Domination in Harary Graphs, Mathematical Notes, 107(2)(2020), 231-237.

[4] K.S. Bagga, L.W. Beineke, W.D. Goddard, M.J. Lipman and R.E.Pippert, A survey of Integrity, Disc. Appl. Math., 37-38(1992), 13-28.

[5] M. Cygan, M. Pilipczuk And R. Skrekovski, Relation between randic index and average distance of trees, MATCH Comm. in Math. and in Com. Chem., 66(2011), 605-612.

[6] T.Cormen, C.E. Leiserson and R.L. Rivest, Introduction to algorithms, The MIT Press, Fourth edition(1990).

[7] H. Frank And I.T. Frisch, Analysis and design of survivable Networks, IEEE Transactions on Communications Technology, 18(5)(1970), 501-519.

[8] J.W.Grossman, F. HaRary. And M. Klawe, Generalized ramsey theory for graphs, x: double star graphs, Disc. Math., 28(3)(1979), 247-254.

[9] I. Gutman, Distance of Thorny Graphs, Publications De L'institut Mathematique Nouvelle serie, 63(77)(1998), 31-36.

[10] T.W. Haynes, S.T. Hedetniemi and P.J. Slater, Fundamentals of domination in graphs, Marcel Dekker Inc., New York,(1998).

[11] T. TURACi And M. OKten, Vulnerability of Mycielski Graphs via Residual Closeness, Ars Combinatoria, 118(2015), 419-427.

[12] M.A. Henning And V. NAicker, Graphs with large disjunctive total domination number, Discrete Math. Theoret. Comput. Sci., 17(2015), 255-282.

[13] M.A. Henning and V. Naicker, Bounds on the Disjunctive Total Domination Number of a Tree, Discus. Math. Graph Theory, 36(2016), 153-171.

[14] M.A. Henning And V. Naicker, Disjunctive total domination in graphs, J. Comb. Optim., 31(3)(2016), 1090-1110.

[15] M.A. Henning And A. Yeo, Total domination in graphs, Springer Monographs in Mathematics, Springer, New York, (2013).

[16] I. Mishrovski, M. Biey and L. Kocarev, Vulnerability of complex Networks, Commun. Nonlinear Sci Numer Simulat., 16(2011), 341-349.

[17] M.V. Modha And K.K. Kanani, On k-cordial labeling of some graphs, British Jour.of Math. and Comp. Sci., 13(3)(2016), 1-7.

[18] S. Nazeer, S.K. Khan, I. Kousar and W. Nazeer,Radio labeling and radio number for generalized caterpillar graphs, J. Appl. Math. and Infor., 34(5-6)(2016), 451-465. 
Disjunctive total domination in some tree networks

[19] F. HARrary,Graph Theory, Addison-Wesley: Boston, MA, USA, (1969).

[20] R. Durgut, H. KutuCu AND T. Turaci, Global distribution center number of some graphs and an algorithm, RAIRO-Operations Research, 53(4)(2019), 1217-1227.

This is an open access article distributed under the Creative Commons Attribution (c) License, which permits unrestricted use, distribution, and reproduction in any medium, provided the original work is properly cited. 